\title{
Relative Positioning Method of Using Single-frequency Model for Formation Flying Missions
}

\author{
Hanyi Shi ${ }^{1}$, Qizi Huangpeng ${ }^{2, a}$, Yuanyuan Qiao ${ }^{3,4}$, Yong $\mathrm{Li}^{3}$, Wenwei Huang ${ }^{4}$ \\ ${ }^{1}$ College of Aerospace Science and Engineering, National University of Defense Technology \\ ${ }^{2}$ College of Five, National University of Defense Technology, Changsha, China \\ ${ }^{3}$ School of Surveying \& Spatial Information Systems, the University of New South Wales, Australia \\ ${ }^{4}$ College of Nine, National University of Defense Technology, Changsha, China \\ ahpqz19911215@163.com
}

Keywords: Satellites in formation flying, lonosphere-free model, Relative positioning, EKF

\begin{abstract}
Use of Global Positioning System (GPS) for space applications, especially in low-earth orbit (LEOs) formation flying missions, has recently attracted more and more attentions. This paper proposes an Extended Kalman Filter (EKF) to increase the accuracy of relative position of LEO formation flying missions. The EKF uses satellite orbital dynamic model, with combination of GPS and carrier phase measurements upon the ionosphere-free model. The carrier phase fuzziness and the position of spacecraft form the state vector, which are predicted in the EKF. Meanwhile, influence of uncertainty of systematic errors is taken into account. The optimal estimation and its modified covariance matrix are derived upon the principle of minimum error covariance. The proposed EKF method is compared with the traditional double difference method in the simulated experiments. The results demonstrate the validity and improvement of the proposed method especially in the long baseline scenarios.
\end{abstract}

\section{Introduction}

GPS has been widely used in the field of mapping and space application, etc., and it was initially applied in land-based positioning. However, in recent years, space application based on GPS, especially in low-earth orbit (LEOs) formation flying missions, has recently attracted more and more attentions [1]. The dispersed structure of the formation flying missions offer better flexibility and redundancy compared to a separate large spacecraft. And it is conducive to a more efficient use of resources and completes the task in less time. A basic problem in formation flying missions is to determine the relative states between the satellites. The double difference method [2] is a commonly used in relative positioning, which can eliminate a series of system error. However, the signal transmission may delay due to ionosphere disturbance that can reduce the estimation precision. A lot of researches are working on it [3][4][5][6] to reduce the disturbance of the ionosphere, but the result is far from satisfaction.

This paper proposes an Extended Kalman Filter (EKF) to increase the accuracy of relative position of LEO formation flying missions. The EKF uses satellite orbital dynamic model, with combination of GPS and carrier phase measurements upon the ionosphere-free model. The carrier phase fuzziness and the position of spacecraft form the state vector, which are predicted in the EKF. Meanwhile, influence of uncertainty of systematic errors is taken into account. The optimal estimation and its modified covariance matrix are derived upon the principle of minimum error covariance.

\section{Modelling of Space System}

Measurement of GPS. For single-frequency receiver which installed in the low orbit satellite, the measurement model for the pseudo range and carrier phase in band L1 is [2]:

$$
\rho_{r}^{s}(t)=r(t)+c\left(\delta t_{r}(t)-\delta t^{s}\left(t-\tau_{r}^{s}(t)\right)\right)+I_{r}^{s}(t)+S_{r}^{s}(t)+\varepsilon_{r P}^{s}(t)
$$




$$
\phi_{r}^{s}(t)=r(t)+c\left(\delta t_{r}(t)-\delta t^{s}\left(t-\tau_{r}^{s}(t)\right)\right)-I_{r}^{s}(t)+S_{r}^{s}(t)+\lambda \varphi_{r}^{s}+\varepsilon_{r \varphi}^{s}(t)
$$

Where $\rho_{r}^{s}$ is measurement of the pseudo range, $t$ is measurement time in GPS, $r$ is the geometric distance between GPS satellites and receiver at time $\left(t-\tau_{r}^{s}\right), \tau_{r}^{s}$ is time of signal transmission, $I_{r}^{s}$ is error in ionosphere, $\delta t_{r}$ is clock correction of receiver, $\delta t^{s}$ is clock correction of GPS satellite, $S_{r}^{s}$ is other system errors, $\varepsilon_{r P}^{s}$ is noise in the pseudo range, $\phi_{r}^{s}$ is distance of the Carrier phase, $\lambda$ is signal wavelength , $\varphi_{r}^{s}$ is the cycle of carrier ambiguous, $\varepsilon_{r \varphi}^{s}$ is noises in the Carrier phase. Usually, the noise in measurement of pseudo range and in the carrier phase are assume as: $E\left(\varepsilon_{r p}^{s}(t)\right)=0, D\left(\varepsilon_{r p}^{s}(t)\right)=\sigma_{P r s}^{2}$.

Ionosphere-free Model of Single-frequency Receiver. Although in equation (1) and (2), the ionosphere error $I_{r}{ }^{s}$ only reflects the first-order effect of the ionosphere, it represents the main effect of the ionosphere. The higher-order of ionosphere effects will cause the effects below millimeter level which can be often ignored. Thus, the Ionosphere-free model of single-frequency Receiver can be obtained according to additive of equation (1) and (2), which remove of the effect ionosphere:

$$
\tilde{\rho}_{r_{-n \text { new }}}^{\mathrm{s}}(t)=0.5\left(\rho_{r}^{\mathrm{s}}(t)+\phi_{r}^{\mathrm{s}}(t)\right)=r_{t}+c\left(\delta t_{r}(t)-\delta t^{s}\left(t-\tau_{r}^{\mathrm{s}}(t)\right)\right)+S_{r, t}^{s}+0.5 \lambda \varphi_{r}^{s}+\varepsilon_{r P_{-} \text {new }}^{s}(t)
$$

Where new noise meets: $E\left(\varepsilon_{r P_{-} \text {new }}^{s}\right)=0, D\left(\varepsilon_{r P_{-} \text {new }}^{s}\right)=(1 / 4)\left(\sigma_{P r s}^{2}+\sigma_{\text {qrs }}^{2}\right)=\sigma_{\text {newrs }}^{2}$.

In equation (3), the satellite clock correction, receiver clock correction and main system error can be eliminated by double difference method. The unknown parameters that need to be estimated include LEO position and fuzziness of carrier phase. The measurement model after double difference processing is:

$$
\nabla \Delta \tilde{\rho}_{r_{-} \text {new }}^{s}(t)=\nabla \Delta r_{t}+0.5 \lambda \nabla \Delta \Delta \varphi_{r}^{s}+\nabla \Delta \varepsilon_{r P_{-} \text {new }}^{s}(t)
$$

Here, $\nabla \Delta$ is double difference operator, $\nabla \Delta \rho_{r, t}^{s}$ is the geometric distance between satellites in double difference, $\lambda$ is wavelength of $L 1$ band, $\nabla \Delta \varphi_{r}^{s}$ is the fuzzy degree of double difference, $\nabla \Delta \varepsilon_{r P_{\text {nnew }}}^{s}$ is noise after double difference processing.

Extended Kalman Filter Model. As for two LEO satellite including a target star and an objective star that installed the single-frequency receiver, assume that the location of main star obtained using the single-point positioning when the location of target star relative is an estimated variables. Suppose the number of other satellites that two satellites can observe at a moment is $n$, the state variable is defined as a $6+n-1$ degree variables: $X_{t}=\left[\begin{array}{llllllll}x & v_{x} & y & v_{y} & z & v_{y} & 0.5 \nabla \Delta \varphi_{r}^{s 1} \cdots 0.5 \nabla \Delta \varphi_{r}^{s n}\end{array}\right]_{t}^{T}$ which composed of the location of objective star and fuzzy degree. Thus, the measurement equation through EKF linearization according to (4) is:

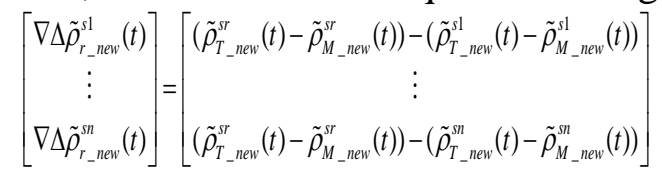

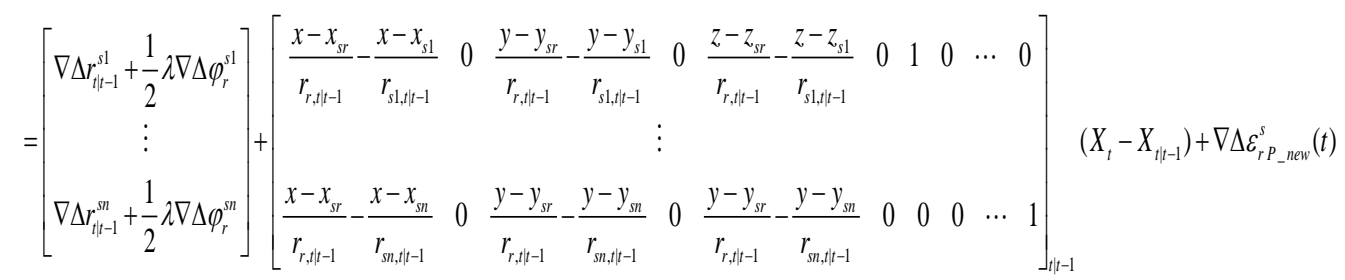

$$
\begin{aligned}
& =h\left(X_{t-t-1}\right)+H_{t t-1} \Delta X_{t}+\nabla \Delta \varepsilon_{t}
\end{aligned}
$$

Where, $\tilde{\rho}_{T_{-} \text {new }}^{\text {sr }}, \quad \tilde{\rho}_{T_{-} \text {new }}^{\text {sk }}, k=1, \cdots, n, k \neq r$ is the ionosphere-free observation of objective star, navigation reference star $r$ and other visible navigation star sk respectively. Here $t$ reperesents moment $t, t \mid t-1$ represents the obtained value of moment $t$ based on moment $t-1 . r_{r, t t-1}$ is the geometric distance between the objective star and navigation reference star, $r_{\text {sk.ttt } 1}, k=1, \cdots, n, k \neq r$ is the geometric distance between the objective star and other visible navigation star. The discrete form of state equation is below.

$$
\Delta X_{t}=\Phi(t, t-1) \Delta X_{t-1}+w_{t}
$$




\section{Relative Positioning Method}

Based on EKF framework, we can estimate the state variable by using predict information as pesudo observation. Defines $\Delta \hat{X}_{t-1}$ as the Optimal estimation based on observation $\nabla \Delta \tilde{\rho}_{r_{-} \text {new, }}^{s 1} \cdots \nabla \Delta \tilde{\rho}_{r_{-} \text {new, } t-1}^{s 1}$. When considering dynamic model prediction error and system measuring error together, the system equation is (7). Here, $\chi$ is dynamic model prediction error and $\kappa$ is system measuring error. $\left\{\kappa_{t}\right\},\left\{\chi_{t}\right\}$ and $\left\{w_{t}, \nabla \Delta \varepsilon_{t}, \Delta X_{0}\right\}$ is mutually exclusive, $\Delta X_{0}, \quad\left\{w_{t}\right\}$ and $\left\{\nabla \Delta \varepsilon_{t}\right\}$ is unrelated. Assume that $Z_{t \mid t-1}=\operatorname{proj}\left\{Z_{t} / Z^{t-1}\right\}$.

$$
\left\{\begin{array}{c}
\Delta X_{t}=\Phi(t, t-1) \Delta X_{t-1}+\chi_{t-1}+w_{t-1} \\
Z_{t}=h\left(X_{t \mid t-1}\right)+H_{t \mid t-1} \Delta X_{t}+\kappa_{t}+\nabla \Delta \varepsilon_{t}
\end{array}\right.
$$

Here, $Z^{t-1}=\operatorname{col}\left\{Z_{0}, Z_{1}, \cdots, Z_{t-1}\right\}$.

In fact, $Z^{t-1}$ and $\nabla \Delta \varepsilon_{0}, \nabla \Delta \varepsilon_{1} \cdots \nabla \Delta \varepsilon_{t-2}, \kappa_{1}, \cdots \kappa_{k-1}$ is related. so:

$$
\begin{gathered}
Z_{t \mid t-1}=\operatorname{proj}\left\{Z_{t} / Z^{t-1}\right\}=\operatorname{proj}\left\{h\left(X_{t \mid t-1}\right)+H_{t \mid t-1} \Delta X_{t}+\kappa_{t}+\nabla \Delta \varepsilon_{t} / Z^{t-1}\right\}=h\left(X_{t \mid t-1}\right)+H_{t \mid t-1} \Delta X_{t \mid t-1}+M_{t} \\
\Delta X_{t \mid t-1}=\operatorname{proj}\left\{\Delta X_{t} / z^{t-1}\right\}=\operatorname{proj}\left\{\Phi(t, t-1) \Delta X_{t-1}+\chi_{t}+w_{t-1} / z^{t-1}\right\}=\Phi(t, t-1) \Delta X_{t-1 \mid t-1}+N_{t}
\end{gathered}
$$

One step of state prediction value is:

$$
\Delta \tilde{X}_{t \mid t-1}=\Delta X_{t}-\Delta X_{t \mid t-1}=\Phi(t, t-1) \Delta X_{t-1}+\chi_{t-1}+w_{t-1}-\left(\Phi(t, t-1) \Delta X_{t-1 \mid t-1}+N_{t}\right)=\Phi(t, t-1) \Delta \tilde{X}_{t-1}+w_{t-1}+\chi_{t}-N_{t}
$$

One step of state prediction variance is:

The new information obtained:

$$
P_{t \mid t-1}=E\left\{\Delta \tilde{X}_{t \mid t-1} \Delta \tilde{X}_{t \mid t-1}{ }^{T}\right\}=\Phi(t, t-1) P_{t-1} \Phi^{T}(t, t-1)+Q_{t-1}+C_{N}(t)
$$

$$
\tilde{Z}_{t \mid t-1}=Z_{t}-Z_{t \mid t-1}=h\left(X_{t \mid t-1}\right)+H_{t \mid t-1} \Delta X_{t}+\kappa_{t}+\nabla \Delta \varepsilon_{t}-\left(h\left(X_{t \mid t-1}\right)+H_{t \mid t-1} \Delta X_{t \mid t-1}+M_{t}\right)=H_{t \mid t-1} \Delta \tilde{X}_{t \mid t-1}+\nabla \Delta \varepsilon_{t}+\kappa_{t}-M_{t}
$$

According to the projection theorem:

New variance is:

$$
\Delta X_{t \mid t}=\operatorname{proj}\left(\Delta X_{t} / Z^{t-1}\right)=\Delta X_{t \mid t-1}+\operatorname{cov}\left\{\Delta \tilde{X}_{t \mid t-1}, \tilde{Z}_{t \mid t-1}\right\} \operatorname{var}^{-1}\left\{\tilde{Z}_{t \mid t-1}\right\} \tilde{Z}_{t \mid t-1}
$$

Gain matrix is:

$$
R_{L}(t)=\operatorname{var}\left\{\tilde{Z}_{t \mid t-1}\right\}
$$

Therefore, the filter value is:

$$
K_{t}=E\left\{\Delta \tilde{X}_{t \mid t-1} \tilde{Z}_{t \mid t-1}^{T}\right\} \operatorname{var}^{-1}\left\{\tilde{Z}_{t \mid t-1}\right\}=E\left\{\Delta \tilde{X}_{t \mid t-1} \tilde{Z}_{t \mid t-1}^{T}\right\} R_{L}(t)^{-1}
$$

$$
\begin{aligned}
& \Delta X_{t \mid t}=\Delta X_{t \mid t-1}+K_{t} \tilde{Z}_{t \mid t-1}=\Delta X_{t \mid t-1}+P_{t \mid t-1} H^{T}{ }_{t \mid t-1} R_{L}{ }^{-1}(t) \tilde{Z}_{t \mid t-1} \\
& =\Delta X_{t \mid t-1}+P_{t \mid t-1} H^{T}{ }_{t \mid t-1}\left(H_{t \mid t-1} P_{t \mid t-1} H_{t \mid t-1}{ }^{T}+R_{t}+C_{M}(t)\right)^{-1} \tilde{Z}_{t \mid t-1}
\end{aligned}
$$

Estimated error is:

$$
\begin{aligned}
& \Delta \tilde{X}_{t \mid t}=\Delta X_{t}-\Delta X_{t \mid t}=\Phi(t, t-1) \Delta X_{t-1}+\chi_{t-1}+w_{t-1}-\left(\Delta X_{t \mid t-1}+P_{t \mid t-1} H^{T}{ }_{t \mid t-1}\left(H_{t \mid t-1} P_{t \mid t-1} H_{t t \mid-1}{ }^{T}+R_{t}+C_{M}(t)\right)^{-1} \tilde{Z}_{t \mid t-1}\right) \\
& =\Phi(t, t-1) \Delta X_{t-1}+\chi_{t-1}+w_{t-1}-\left(\Phi(t, t-1) \Delta X_{t-1 \mid t-1}+N_{t}+P_{t \mid t-1} H^{T}{ }_{t \mid t-1}\left(H_{t \mid t-1} P_{t \mid t-1} H_{t \mid t-1}{ }^{T}+R_{t}+C_{M}(t)\right)^{-1} \tilde{Z}_{t \mid t-1}\right) \\
& =\Phi(t, t-1) \Delta \tilde{X}_{t-1}+\chi(t-1)-N_{t}+w_{t-1}-P_{t \mid t-1} H^{T}{ }_{t \mid t-1} R_{L}(t)^{-1} \tilde{Z}_{t \mid t-1}
\end{aligned}
$$

Prediction variance are as follow:

$$
\begin{aligned}
& P_{t \mid t}=E\left\{\Delta \tilde{X}_{t \mid t} \Delta \tilde{X}_{t \mid t}^{T}\right\}=\Phi(t, t-1) P_{t-1 \mid t-1} \Phi(t, t-1)^{T}+Q_{t}+C_{N}(t)-P_{t \mid t-1} H_{t \mid t-1}^{T} R_{L}(t)^{-T} H_{t \mid t-1} P_{t \mid t-1}{ }^{T} \\
& =P_{t \mid t-1}-P_{t \mid t-1} H_{t \mid t-1}{ }^{T} R_{L}(t)^{-T} H_{t \mid t-1} P_{t \mid t-1}{ }^{T}
\end{aligned}
$$

We can see that the system error in state eqaution shows in prediction state $\Delta X_{t \mid t-1}$ and prediction variance $P_{t \mid t-1}$. The system error of measurement equation is in new information $\tilde{Z}_{t \mid t-1}$ and covariance $R_{L}$.

\section{Simulation Experiment}

First, we determine the required simulation parameters: the order of earth's gravity field model is 30. The visible shielding angle of navigation star is 5 degree. The standard deviation of fuzzy degree is $5 \times 10^{8}$ week. The altitude of two satellites orbit is $300 \mathrm{~km}$ and the baseline between them is 100 $\mathrm{km}$. The simulation condition is: the satellite orbit is in relatively low altitude, and influenced by 
ionosphere a lot. Compare proposed method with RTKLIB in calculating two satellite's baselines. RTKLIB use the classical double difference method to obtain the relative position in the 'moving base station' mode. Using pseudo range and double difference carrier phase observation value to get the relative position of the target star by least square method, and then use LAMBDA algorithm to get the fuzzy degree and correct the calculation results.

The relative position estimation error of objective star calculated by RTKLIB is shown in Table 1 . It describes the error in mode A 'Fix and Hold' can gets the result by fix the value and get fuzzy degree. The result in mode B 'Instantaneous'. It get every moment's fuzzy degree through correction. Usually, if the observation result obtained by double difference processing is not affected by system error, the mode $\mathrm{A}$ is not affected by the quality of the data with time measuring. It can defined as a common model which works well. In this simulation experiment, measure the accuracy by the three axis estimation mean square root, the accuracy of the results in the reletive positioning method is increased by $94.19 \%$ compared to RTKLIB in mode A. The accuracy of the results is increased by 78.08\% compared to RTKLIB in mode B. It seens that proposed method enchances the accuracy compared to classical method a lot.

Table 1 Comparison of different methods

\begin{tabular}{cccc}
\hline Method & Precision in x axi & $\begin{array}{c}\text { Precision in } \mathrm{y} \\
\text { axi }\end{array}$ & Precision in z axi \\
\hline Mode A & 3.839 & 5.043 & 7.465 \\
Mode B & 0.515 & 0.439 & 2.508 \\
Reletive Positioning & 0.374 & 0.398 & 0.161 \\
\hline
\end{tabular}

\section{Conclusion}

Aimed at the single-frequency GPS receiver which has the advantages of low cost and light in weight, this paper puts forward a method of using single-frequency ionosphere-free model for formation flying missions of low earth orbit satellite relative position calculation. The method in the framework of the EKF, combined the estimate of fuzzy degree and carrier phase toghter. Meanwhile, proposed method taking the effects of other system error into account, coreect the noise covariance matrix and get optimal estimation of the minimum variance. In the simulation experiment, the relative positioning method is compared with the results obtained in RTKLIB in different processing classical mode. It show that the proposed method could greatly improve the precision of relative positioning of the target star.The future work is mainly to study the higher precision relative positioning method in UKF theory and the research of how to improve the absolute positioning based on single frequency GPS receiver .

\section{Acknowledgements}

This work is supported by the Chinese National Science Foundation grant 60974124 and 61004081.

\section{References}

[1] Simone D’Amico (2010), Autonomous formation flying in Low Earth Orbit, Published by Ridderprint BV, Delft

[2] Gleason Scott (2009), GNSS Applications and Methods, Artech House press, Norwood, MA

[3] B Hofmann-Wellenhof, H Lichtenegger, E Wasle (2007), GNSS-global navigation satellite systems, Springer-Verlag press, New York, 127-218.

[4] Chang-Moon Lee, Kwan-Dong Park (2011), Generation of Klobuchar ionosphere error model coefficients using fourier series and accuracy analysis, Journal of Astronomy and space science, 28(1): 71-77 
[5] H.A.Marques, J.F.G.Monico, M.Aquino (2011), RINEX_HO: second- and third-order ionospheric corrections for RINEX observation files, GPS solution, 15(3): 305-314

[6] T.P. Yunck, Coping with the atmosphere and ionosphere in precise satellite and ground positioning, in: A. Valance-Jones (Ed.), Environmental Effects on Spacecraft Trajectories and Positioning, AGU Monograph, 1993. 